\title{
Multiplexed frequency spectrum analyzer instrumentation for the characterization of multiple QCM-based biosensors
}

\author{
$\underline{\text { Atman Jbari }}{ }^{1}$, Larbi Bellarbi ${ }^{2}$, Nadia Zine ${ }^{4}$, Christopher A. Mills ${ }^{4}$, \\ Josep Samitier ${ }^{3,4}$, Abdelhamid Errachid ${ }^{3,4}$ \\ ${ }^{1,2}$ Laboratoire de Génie Electrique, ENSET-RABAT, ${ }^{3}$ Universitat de Barcelona,Martí $i$ \\ Franquès, 1, 08028, Barcelona, ${ }^{4}$ Institut de Bioenginyeria de Catalunya (IBEC), Parc Científic \\ de Barcelona, C/ Josep Samitier 1-5, 08028 Barcelona \\ E-mail : atmjbari@yahoo.fr, aerrachid@pcb.ub.es
}

\begin{abstract}
In this contribution, we present novel multiplexed frequency spectrum analyzer instrumentation to extract operational parameters and completely characterize the frequency response of an array of quartz crystal microbalance sensors. The effectiveness of the proposed instrumentation is proven by experimental measurements over a range of frequencies.
\end{abstract}

\section{Introduction}

The bio-detection or measure of nano-parameters represent an important way in scientific investigations and is applied in various domains: ADN Hybridizing, protein-protein interaction, biosensors development, corrosion and absorption studies, electrochemical deposition, dithienothiophene-based polymers [1-6].

Several types of sensors are used; the particularity of piezoelectric sensors is to traduce the variation of mass to the variation of resonant frequency with high sensitivity [7]. Therefore, the microbalance technique is a good practical motivation in biosensors domain and their applications.

Several commercial microbalances (for example QCA922, QCM100, QCM200) measure only the series frequency and resistance at resonance. In this contribution, we present the development of instrumentation that gives a complete characterization of a set of quartz sensors and the soft system for acquisition and treatment.

This paper is organized as follows: In section 2, we present the principles of microbalance, quartz model, and measurement methods. In section $\mathbf{3}$ we trait and we choice the electronic component of different blocs of the proposed instrumentation system. Section
4 present the set of measures effected in order to validate our system. Finally, we conclude on global performances and advantages of our system.

\section{Quartz microbalance principles}

\subsection{Microbalance principles}

The quartz crystal microbalance (QCM) exploits the phenomenon of piezoelectricity present in some natural and artificial materials. The direct piezoelectric effect describes the property of a dielectric material to create a voltage, or variation of potential, when it is subjected to a mechanical deformation. As a consequence, it is possible to use this effect to measure several physical parameters, such as acceleration, pressure etc., which can be used for a piezoelectric sensor. The inverse piezoelectric effect, or electrostriction, describes the opposite effect where the piezoelectric material deforms under an applied electrical potential.

The resonant frequency of AT cut quartz crystals (low sensitivity to temperature) changes as a function of mass deposited on its surface, and can be represented by the Sauerbrey equation [8]:

$$
\Delta F=-\frac{2 F_{2}}{A \sqrt{\mu \rho_{Q}}} \Delta m
$$

where, $\Delta \mathbf{F}$ : frequency variation due to mass loading, $\mathbf{F}$ : quartz crystal resonant frequency, $\Delta \mathbf{m}$ : mass variation, $\boldsymbol{\rho}_{\mathbf{Q}}$ : density of quartz, $\boldsymbol{\mu}$ : shear modulus, $\mathbf{A}$ : piezoelectric active area.

\subsection{Quartz microbalance sensors}

The equivalent electrical circuit for the quartz crystal is presented in figure $\mathbf{1}$. 
Published in: 2007 International Conference on Sensor Technologies and Applications, SENSORCOMM 2007, Proceedings, art. no. 4394960 , pp. 436-440

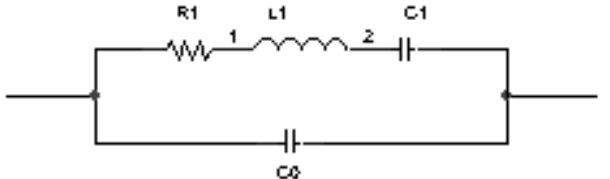

Figure 1 : Equivalent electrical circuit of the QCM.

where, $\mathbf{C}_{\mathbf{0}}$ : static capacity, $\mathbf{R}_{\mathbf{1}}$ : Resistance (viscosity), $\mathbf{C}_{\mathbf{1}}$ : dynamic capacity, $\mathbf{L}_{\mathbf{1}}$ : Inductance of quartz (energy gain)

The series resonance frequency, $\mathbf{F}_{\mathrm{s}}$, is:

$$
F_{s}=\frac{1}{2 \pi \sqrt{L_{1} C_{1}}}
$$

$\mathbf{F}_{\mathbf{s}}$ changes as a function of mass and elasticity, and measurement of Fs allows the quartz crystal parameters to be determined.

Complex admittance, $\mathbf{Y}$, can be written as:

$$
Y(j \omega)=j C_{0} \omega+\frac{1}{R_{1}+j L_{1} \omega+\frac{1}{j C_{1} \omega}}
$$

The conductance, $\mathbf{G}=\mathbf{R e}(\mathbf{Y}(\mathbf{j} \boldsymbol{\omega}))$, and suceptance, $\mathbf{B}=\mathbf{I m}(\mathbf{Y}(\mathbf{j} \omega))$, are related by:

$$
\left(G-\frac{1}{2 R_{1}}\right)^{2}+\left(B-\omega C_{0}\right)^{2}=\left(\frac{1}{2 R_{1}}\right)^{2}
$$

These equations can be used to model the frequency relationship of a crystal. For example, figures 2 and 3 model a quartz crystal with, $\mathbf{C}_{\mathbf{0}}=\mathbf{2 2} \mathbf{p F} ; \mathbf{R}_{\mathbf{1}}=\mathbf{1 0 0} \Omega$;

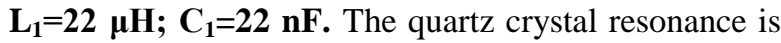
therefore characterized by its resonance frequency (9.95 MHz, $10.2 \mathrm{mS}, 12^{\circ}$ ).

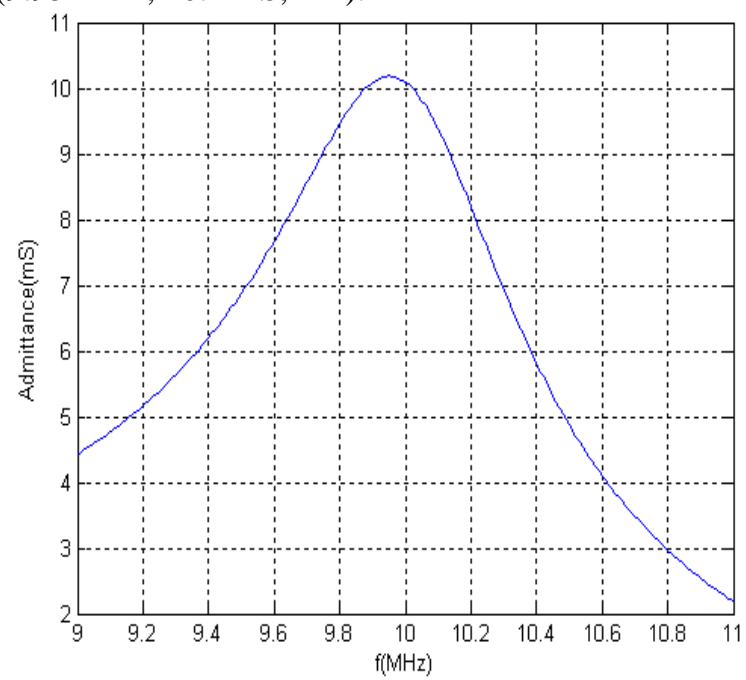

Figure 2 : Quartz Modulus Admittance vs. frequency.

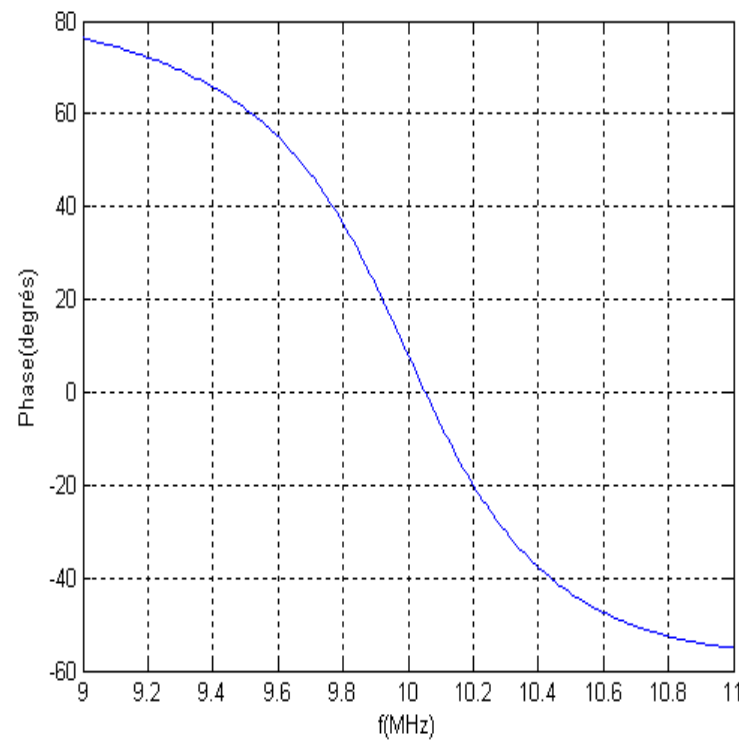

Figure 3 : Quartz phase Admittance vs. Frequency

\subsection{Measurement methods:}

There are two possible measurement methods:

i) Active method:

The quartz crystal is placed in oscillator circuit (Miller or Collpits). The oscillations are maintained by a reaction loop and the frequency fluctuations represent a variation of mass, elasticity or viscosity, which is measured by a frequency-meter device. This principle is simple but the extraction of the crystals parameters is impossible.

\section{ii) Passive method:}

The quartz crystal receives a sinusoidal excitation (voltage or current). A network analyzer estimates and represents the equivalent admittance and capacitance $\left(\mathrm{L}_{1}, \mathrm{C}_{1}\right)$ values.

\section{Microbalance instrumentation}

The proposed microbalance instrumentation must have the following characteristics:

a) Multiple sensors analysis

b) Frequency measurement up to $100 \mathrm{MHz}$

c) Complete characterization of sensor (including resonant frequency, resistance and admittance)

d) Possibility to effect frequency sweep or single frequency analysis.

e) Frequency resolution $\leq 0.1 \mathrm{~Hz}$

f) Time sweep $\leq 1 \mathrm{~s}$

g) Low electrical power consumption. 
Published in: 2007 International Conference on Sensor Technologies and Applications, SENSORCOMM 2007, Proceedings, art. no. 4394960 , pp. 436-440

\subsection{Microbalance instrumentation function}

Figure 4 illustrates the functional diagram for the proposed microbalance instrumentation. A DDS circuit, controlled by a reference oscillator, generates the sinusoidal excitations to a sensor selected by an analog switch. An instrumentation amplifier receives the voltage from the quartz sensor and presents it to the input of a Amplitude/Phase detection block. After analog to digital (A/D) conversion, a computer acquires the signal through an Input/Output USB interface.

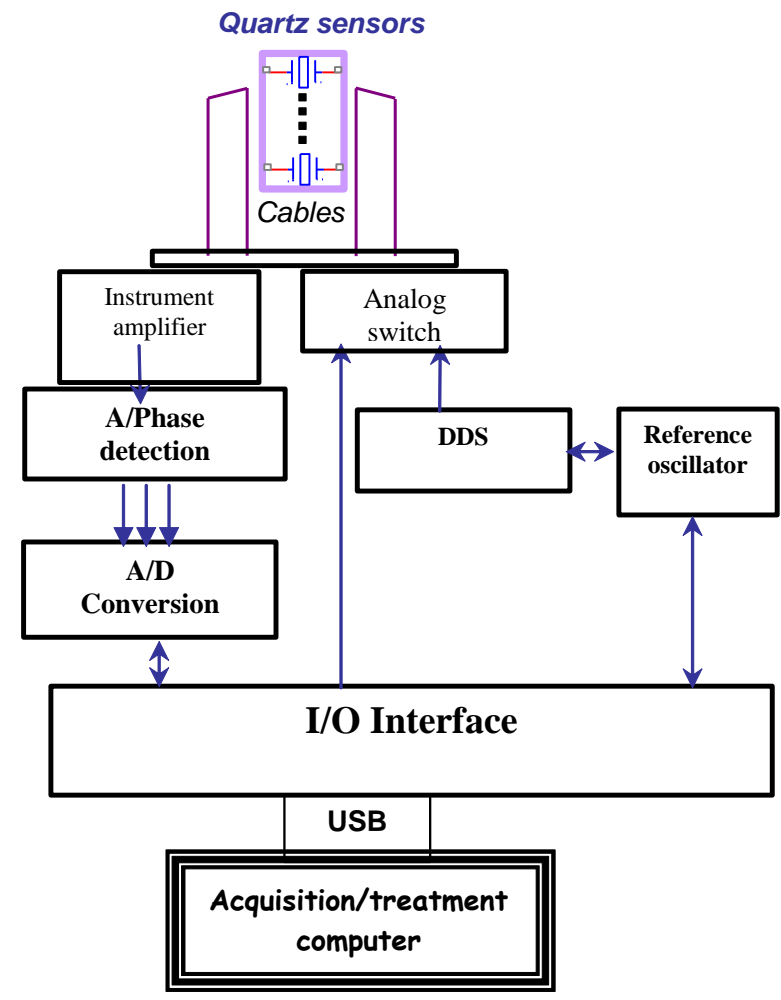

Figure 4 : Microbalance instrumentation functional diagram.

\subsection{Electronics}

The electronics are composed of an instrumentation amplifier and a measurement block: amplitude, current and phase. The amplifier must isolate the required sensor and measure the measurement block. For measurement block, we use the synchronous detection method represented in figure 5.

$\mathbf{v}_{\mathbf{r e}}$ : reference voltage, $\mathbf{v}_{\mathbf{e}}$ : sensor excitation voltage, $\mathbf{v}_{\mathbf{q}}$ : sensor voltage, $\mathbf{i}_{\mathbf{q}}$ : sensor current, $\mathbf{v}_{\mathbf{i}}$ : voltage of reference resistance $R_{r}$ (current image iq), $\mathbf{v}_{\mathbf{m} 1}, \mathbf{v}_{\mathbf{m} 2}, \mathbf{v}_{\mathbf{m} \mathbf{3}}$ : outputs of multipliers $\mathrm{M}_{1}, \mathrm{M}_{2}$ et $\mathrm{M}_{3}, \mathbf{V}_{\mathbf{f 1}}$, $\mathbf{V}_{\mathbf{f} 2}, \mathbf{V}_{\mathbf{f} 3}$ outputs of filters $\mathrm{F}_{1}, \mathrm{~F}_{2}$ et $\mathrm{F}_{3}$.

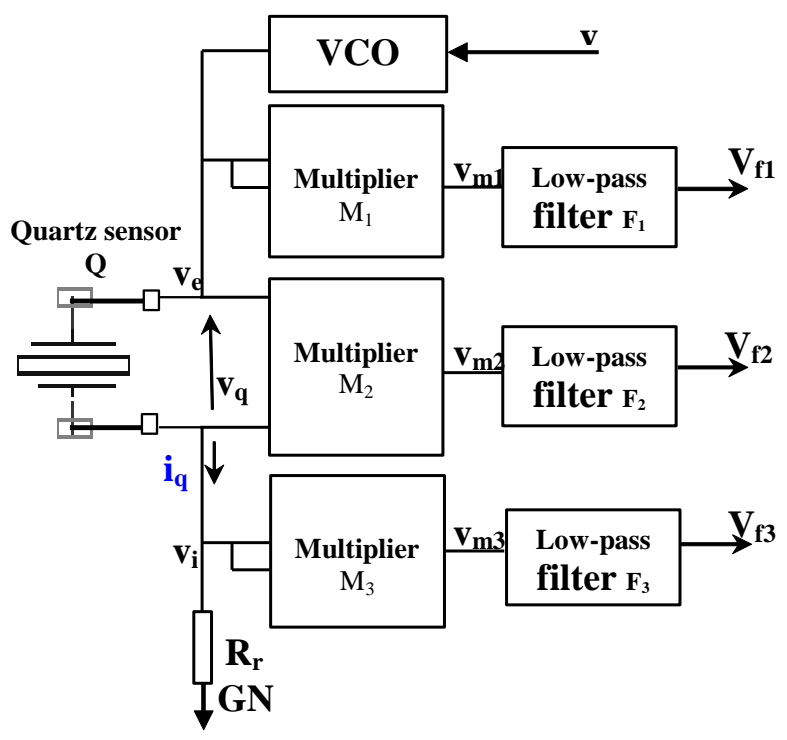

Figure 5: Instrumentation block diagram

Voltage expressions :

Excitation voltage: $\mathrm{v}_{\mathrm{e}}(\mathrm{t})=\mathrm{v}_{\mathrm{e}} \cos \omega_{\mathrm{e}} \mathrm{t}=\mathrm{v}_{\mathrm{e}} \cos 2 \pi \mathrm{f}_{\mathrm{e}} \mathrm{t}$

Resistance voltage: $v_{i}(t)=v_{i} \cos \left(\omega_{e} t+\Phi_{i}\right)=R_{r} i_{q}(t)$

Multiplier outputs :

$\mathrm{M}_{1}: \mathrm{v}_{\mathrm{m} 1}(\mathrm{t})=\mathrm{K}_{\mathrm{m} 1} \mathrm{v}_{\mathrm{e}}^{2}(\mathrm{t})=\mathrm{K}_{\mathrm{m} 1} \mathrm{~V}_{\mathrm{e}}^{2}\left(1+\cos 2 \omega_{\mathrm{e}} \mathrm{t}\right) / 2$

$\mathrm{M}_{2}: \mathrm{v}_{\mathrm{m} 2}(\mathrm{t})=\mathrm{K}_{\mathrm{m} 2} \mathrm{v}_{\mathrm{e}}(\mathrm{t}) \mathrm{v}_{\mathrm{i}}(\mathrm{t})=\mathrm{K}_{\mathrm{m} 2} \mathrm{~V}_{\mathrm{e}} \mathrm{V}_{\mathrm{i}}\left(\cos \Phi_{\mathrm{i}}+\cos \right.$ $\left.\left(2 \omega_{\mathrm{e}} \mathrm{t}+2 \Phi_{\mathrm{i}}\right)\right) / 2$

$\mathrm{M}_{3}: \mathrm{v}_{\mathrm{m} 3}(\mathrm{t})=\mathrm{K}_{\mathrm{m} 3} \mathrm{~V}_{\mathrm{i}}^{2}(\mathrm{t})=\mathrm{K}_{\mathrm{m} 3} \mathrm{~V}_{\mathrm{i}}^{2}\left(1+\cos \left(2 \omega_{\mathrm{e}} \mathrm{t}+2 \Phi_{\mathrm{i}}\right)\right)$ 12

Filter outputs :

$\mathbf{F}_{1}: \mathrm{V}_{\mathrm{f} 1}(\mathrm{t})=\mathrm{K}_{\mathrm{m} 1} \mathrm{~V}_{\mathrm{e}}^{2} / 2$

$\mathbf{F}_{2}: \mathrm{V}_{\mathrm{f} 2}(\mathrm{t})=\mathrm{K}_{\mathrm{m} 2} \mathrm{~V}_{\mathrm{e}} \mathrm{V}_{\mathrm{i}} \cos \Phi_{\mathrm{i}} / 2$

$\mathbf{F}_{3}: \mathrm{V}_{\mathrm{f} 3}(\mathrm{t})=\mathrm{K}_{\mathrm{m} 3} \mathrm{~V}_{\mathrm{i}}^{2} / 2$

The quartz impedance, $Z$, is,

$$
Z(j \omega)=\frac{V_{q}(j \omega)}{I_{q}(j \omega)}=\frac{R_{r}\left(V_{q}(j \omega)-V_{i}(j \omega)\right)}{V_{i}(j \omega)}
$$

Then we have,

$$
Z(j \omega)=\frac{R_{r} V_{e}(j \omega)}{V_{i}(j \omega)}-R_{r}
$$

$$
\begin{aligned}
& R_{e}(Z)=R_{r}\left(\frac{V_{e}}{V_{i}} \cos \phi_{i}-1\right) \\
& \operatorname{Im}(Z)=R_{r} \frac{V_{e}}{V_{i}} \sin \phi_{i}
\end{aligned}
$$


Published in: 2007 International Conference on Sensor Technologies and Applications, SENSORCOMM 2007,

Proceedings, art. no. 4394960 , pp. 436-440

As the common rejection ratio rejection mode must be $\geq 60 \mathrm{~dB}$, the frequency band analysis $\leq 100 \mathrm{MHz}$ and the slew rate, $\mathrm{Sr}>628 \mathrm{~V} / \mu \mathrm{s}$, we have chosen AD8129 (instrumentation amplifier), AD835 (multiplier), MAX038 (reference oscillator), AD9850 (DDS), AD 7655 (14bits Analog to digital converter), NI-USB 6251(USB-I/O interface) for use. The DDS AD9850 allows frequency control at $\mathbf{0 . 0 2 9 1} \mathbf{H z}$ resolution (reference: $125 \mathrm{MHz}$ ).

\section{Low-pass filter:}

In order to reject the harmonic frequencies, $\mathbf{2} \omega_{\mathrm{e}}$, we use a second order low-pass filter, RC. This filter assures stability and suppresses waves caused by fast variation of the amplitude. In addition, the filter must be rapid and able to allow rapid frequency sweeps. We will work at frequencies of $\mathbf{1 M H z}$, therefore we use $\mathrm{R}$ $=10 \mathrm{k} \Omega ; \mathrm{C}=2.2 \mathrm{nF}$.

\section{Results and discussion}

In order to demonstrate the effectiveness of the proposed instrumentation unit, several experiments are presented using a $9 \mathrm{MHz}$ quartz crystal (figures 6-9). In all measurements, the voltage excitation is sinusoidal with an amplitude, $\mathbf{V e}=\mathbf{1} \mathbf{V}$. The square wave reference signal trigger, $\mathbf{v}_{\mathbf{t}}$, is also given which controls the linear sweep frequency.

Note: $\mathbf{F}_{\min }$ : Minimal frequency analyzer, $\mathbf{F}_{\max }$ : Maximal frequency analyzer $\left(\Delta \mathbf{F}=\mathbf{F}_{\max }-\mathbf{F}_{\min }\right), \mathbf{F}_{\text {res }}$ : quartz resonant frequency, Ts : Seep period which corresponds to the signal period $\mathbf{v}_{\mathbf{t}}$.

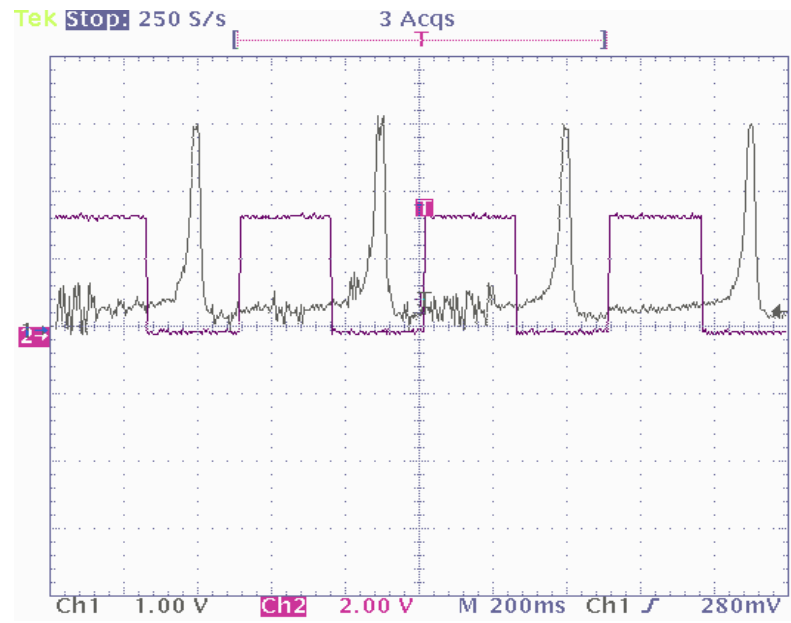

Figure 6: $F_{\min }=8.0 \mathrm{MHz}, F_{\max }=9.0 \mathrm{MHz}, \Delta \mathrm{F}=1 \mathrm{MHz}$, $\mathrm{Ts}=500 \mathrm{~ms}$. $\mathrm{F}_{\text {res }}=8,98 \mathrm{MHz}$. The resonant peak can be clearly seen in this time period.

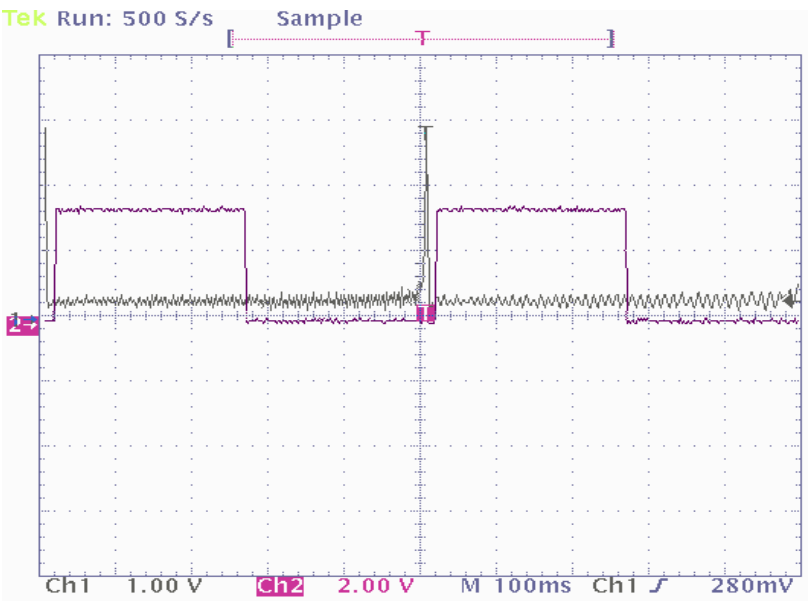

Figure 7: Increasing frequency, $\mathrm{F}_{\min }=8.9 \mathrm{MHz}, \mathrm{F}_{\max }=$ $8.99 \mathrm{MHz} ; \mathrm{Ts}=500 \mathrm{~ms}$. The resonant peak can be clearly seen in this time period near to ascendant trigger pulse.

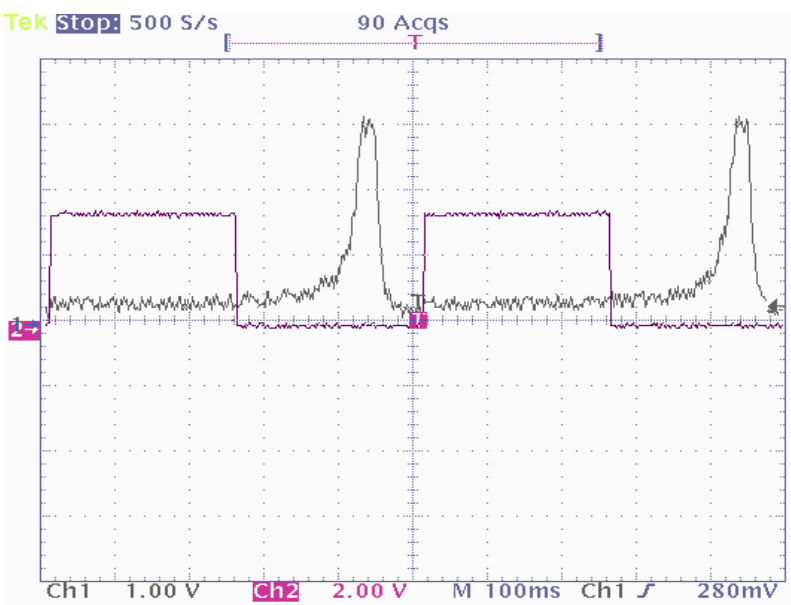

Figure 8: Reducing the frequency, $F_{\min }=8.96 \mathrm{MHz}$, $\mathrm{F}_{\max }=8.99 \mathrm{MHz}, \mathrm{T}_{\mathrm{s}}=500 \mathrm{~ms}$.

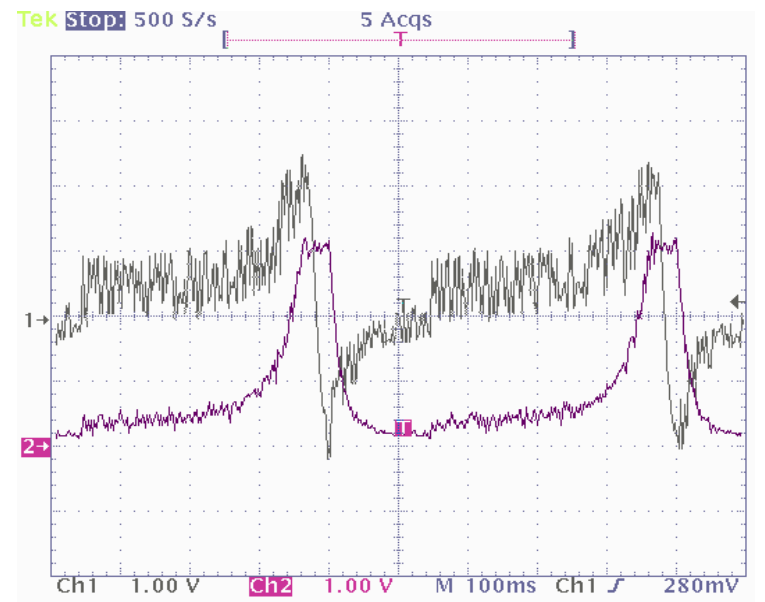

Figure 9: Amplitude and Phase, $F_{\min }=8.95 \mathrm{MHz}, \mathrm{F}_{\max }$ $=8.99 \mathrm{MHz}, \Delta \mathrm{F}=1 \mathrm{MHz}, \mathrm{Ts}=500 \mathrm{~ms}$. 
Figure 9 shows the amplitude and phase signals. At resonance, the amplitude can be seen to peak whereas the phase falls rapidly before recovering. This demonstrates the change in impedance behavior.

These measurements present the localization of resonant peak for several sweep intervals and validate our technique which can be performed by using an acquisition interface and treatment system presented in the following section.

\section{Acquisition and treatment software}

To collate and treat the data, QCMicrobalance software has been developed which acquires instrumentation signals and describes the functions given in figure $\mathbf{1 0}$.

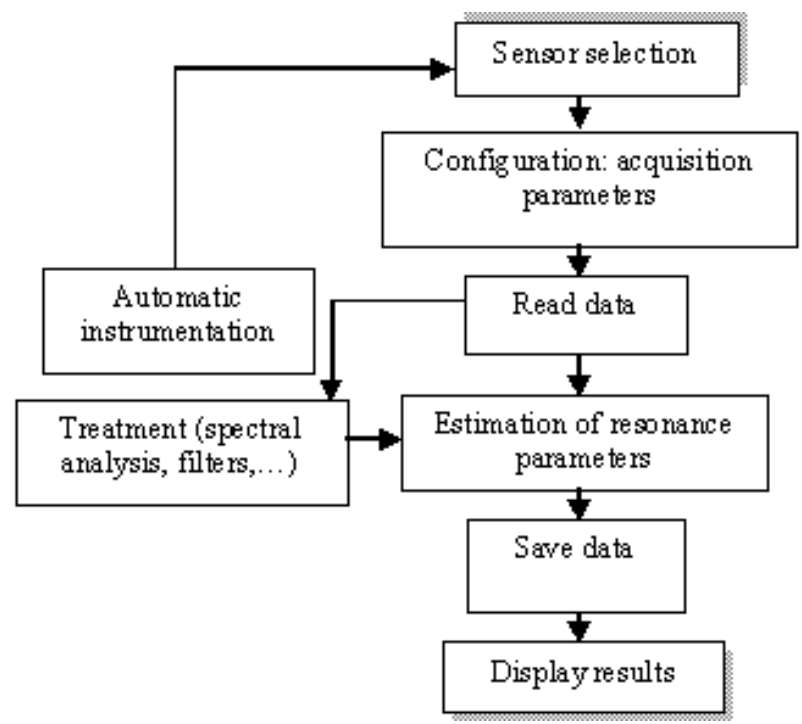

Figure 10: Acquisition and treatment functions

The automatic instrumentation function ensures that the measurements occur with temporal confinements. It allows us to program the start/stop commands and define the measurement period.

\section{Conclusion}

The proposed novel instrumentation allows the measurement of the parameters that completely determine the quartz crystal behavior in the frequency domain. It can control an array of sensors allowing the possibility of simultaneous experiments or simultaneous characterization of several dependent parameters. This microbalance requires an power supply of $+\mathbf{5 V} / \mathbf{- 5} \mathrm{V}$. The amplitude of the quartz excitation voltage is $1 \mathrm{~V}$.

In addition, the use of digital synthesizer circuit assures a precise analysis of frequency. This microbalance, with data exploitation and treatment software, constitute a performance tool for all experiments requiring the use of quartz crystal microbalance sensors.

\section{References :}

[1] A. Jbari, L. Bellarbi, A. Errachid, Développement de Biocapteurs Basés sur la microbalance à quartz modifiée par le transfert du film Langmuir-Blodgett d'immunoglobuline G/amphiphile, Congrès de biotechnologie-Settat 06 mai 2005.

[2] C. A. Mills, J. Beeley, C. Wyse, D. R. S. Cumming, A. Glidle, J. M. Cooper, Polymer-based micro-sensor paired arrays for the determination of primary alcohol vapors, Sens. Act. B (in press)

[3] C. A. Mills, K. T. C. Chai, M. J. Milgrew, A. Glidle, J. M. Cooper, D. R. S. Cumming, A Multiplexed Impedance Analyzer for Characterizing Polymer-Coated QCM Sensor Arrays, IEEE Sens. J. 6 (2006) 996-1002

[4] J. M. Beeley, C. Mills, P. A. Hammond, A. Glidle, J. M. Cooper, L. Wang, D. R. S. Cumming, All-digital interface ASIC for a QCM-based electronic nose, Sens. Act. B 103 (2004) 31-36

[5] I.A. Marques de Oliveira, M.B. Santos, S. Rodriguez, M.T.S.R. Gomes, R. Eritja, J. Samitier and A. Errachid, First characterization of a biosensor for large DNA molecules using quartz crystal microbalance and impedance spectroscopy, Transducers'04 \& Eurosensors XXI, 10-14, June, Lyon France, 2007

[6] S. A. Rodriguez-Segui, I. Bucior, M. M. Burger, J.Samitier, A.Errachid and X. Fernandez-Busquets, Aplication of a bio-QCM to study carbohydrates selfinteraction in presence of calcium, Transducers'04 \& Eurosensors XXI, 10-14, June, Lyon France, 2007

[7] F. Soavi,C. Arbizzani and M. Mastragostion, Quart crystal impedance and EQCM measurements applied to dithienothiophene-based polymers. Chem. Phys.,2000 pp- 2993-2998.

[8] S. J.Martin , V. E. Granstaff, and G. C. Frye. Characterization of a Quartz Crystal Microbalance with Simultaneous Mass and Liquid Loading. Sandia National Laboratories, Albuquerque, New Mexico 87185 .Sauerbrey . 